\title{
The pion mass in finite volume to two loops
}

\section{Christoph Haefeli*}

Institut für theoretische Physik

Universität Bern

Sidlerstr. 5, 3012 Bern

E-mail: haefeli@itp.unibe.ch

We evaluate the pion mass in finite volume to two loops within Chiral Perturbation Theory. The results are compared with a recently proposed extension of the asymptotic formula of Lüscher. We find that contributions, which were neglected in the latter, are numerically very small at the two-loop level.

XXIIIrd International Symposium on Lattice Field Theory

25-30 July 2005

Trinity College, Dublin, Ireland

*Speaker. 


\section{Introduction}

Numerical simulations with lattice QCD are bound to rather small lattice volumes when determining the hadron spectrum and other low energy parameters in QCD. The computed observables show a volume dependence and a thorough understanding of these effects is important for a correct interpretation of numerical data. We report on recent progress related to analytical finite volume studies in case of the pion mass.

A long time ago Lüscher established an asymptotic formula [1] which relates the size dependence of the pion mass $M_{\pi}$ with the $\pi \pi$-forward scattering amplitude $\mathscr{F}_{\pi}(v)$,

$$
M_{\pi L}-M_{\pi}=-\frac{3}{16 \pi^{2} M_{\pi} L} \int_{-\infty}^{\infty} d y \mathscr{F}_{\pi}(\mathrm{i} y) e^{-\sqrt{M_{\pi}^{2}+y^{2}} L}+O\left(e^{-\sqrt{3 / 2} M_{\pi} L}\right),
$$

with $M_{\pi L}$ the pion mass in finite volume and where the constraint $M_{\pi} L \gg 1$ is assumed. The contributions in the low energy region are enhanced, where the $\pi \pi$-scattering amplitude is represented very accurately through its chiral representation $[2,3]$. At leading order in the chiral counting the forward scattering amplitude assumes to be a constant, and the integral simplifies to a modified Bessel function of the second kind. When Colangelo and Dürr evaluated subleading finite volume effects with Lüscher's formula, they observed numerically enhanced corrections with respect to the leading order [4]. These investigations show a clear necessity to go beyond leading order calculations in order to have the finite volume effects of the pion mass under control. Furthermore, as the derivation of eq. (1.1) takes into account only exponential contributions of the order $\exp \left(-M_{\pi} L\right)$ and systematically drops those of the order $\exp \left(-\sqrt{3 / 2} M_{\pi} L\right)$, the question arises, whether the missed terms might turn out to be numerically relevant. The same question also concerns the asymptotic formulae of decay constants [5]. As we will show, a full two-loop calculation of the pion mass in finite volume ChPT clarifies these open points.

The two-loop calculation appears to be interesting also for its own right. To date, a number of finite volume calculations have been performed at one-loop order [6], but as far as we know two-loop calculations have only been performed for the quark condensate [7] and for low-energy observables in a closely related field [8]. As finite volume effects occur first at the one-loop level, only a two-loop calculation - or alternatively if existing, an asymptotic formula à la Lüscher leads to a better understanding of the convergence behaviour of the perturbative expansion.

\section{ChPT in finite volume}

Chiral Perturbation Theory (ChPT) is the effective theory for QCD at low energies. It is nowadays a mature field which has been applied successfully for a variety of phenomena, in particular in the meson sector. For an introduction and a current status of the field, we refer to Ref. [9, 10].

The effective framework is still appropriate, when the system is enclosed by a box of size $V=L^{3}$. We refer to the literature for the foundations $[11,12,13]$ and a recent review [14]. Here, we only remind of the fundamental results which guided the present calculation: the volume has to be large enough, such that ChPT can give reliable results, $2 F_{\pi} L \gg 1$. The perturbative calculation is bound to the value of the parameter $M_{\pi} L$. Whether it exhibits to be large $\left(M_{\pi} L \gg 1\right.$, "p-regime") or small $\left(M_{\pi} L \lesssim 1\right.$, " $\varepsilon$-regime") implies a different power counting. However, in both cases the 
effective Lagrangian is the same as in the infinite volume. Here, we only cover the " $p$-regime", where the system is distorted mildly and the only change brought about by the finite volume is a modification of the pion propagator due to the periodic boundary conditions of the pion fields

$$
G\left(x^{0}, \mathbf{x}\right)=\sum_{\mathbf{n} \in \mathbb{Z}^{3}} G_{0}\left(x^{0}, \mathbf{x}+\mathbf{n} L\right)
$$

with $G_{0}(x)$ the propagator in infinite volume.

\section{Pion in finite volume}

The pion mass in finite volume is defined by the pole equation

$$
G\left(\hat{p}_{L}\right)^{-1}=0, \quad \text { for } \quad \hat{p}_{L}=\left(i M_{\pi L}, \mathbf{0}\right),
$$

where $G\left(p^{0}, \mathbf{p}\right)^{-1}$ is the Fourier transform of the connected correlation function

$$
\begin{aligned}
\left\langle\pi^{1}(x) \pi^{1}(0)\right\rangle_{L} & =L^{-3} \sum_{\mathbf{p}} \int \frac{d p^{0}}{2 \pi} e^{i p x} G\left(p^{0}, \mathbf{p}\right), \\
G\left(p^{0}, \mathbf{p}\right)^{-1} & =M^{2}+p^{2}-\Sigma_{L}\left(p^{2}\right),
\end{aligned}
$$

with $\Sigma_{L}\left(p^{2}\right)$ the self-energy in finite volume and $M^{2}$ the pion mass in the chiral limit in infinite volume. A determination of the pion mass amounts thus to an evaluation of the self-energy in a loop expansion. At one-loop order the finite volume corrections have been evaluated in [11]. Here, we discuss the main steps which guided the two-loop calculation. A detailed derivation of the results will be given elsewhere [15]. It is convenient to write the pion mass in finite volume to two loops in the following manner,

$$
\begin{aligned}
M_{\pi L}^{2} & =M_{\pi}^{2}-\Sigma^{(1)}-\Sigma^{(2)}, \\
M_{\pi}^{2} & =M^{2}-\Sigma^{(0)},
\end{aligned}
$$

where $\Sigma^{(r)}, r=0,1,2$, denote the contribution of the self-energy with $r$ propagators in finite volume with non-vanishing vector $\mathbf{n}$ (cf. eq. (2.1)). These terms shall be discussed in some detail.

\subsection{Self-energy to 0'th order: $\Sigma^{(0)}$}

The contributions to $\Sigma^{(0)}$ are volume independent by definition and merely renormalize the pion mass, cf. eq. (3.3). A detailed discussion of this calculation can be found in [2], with which we agree.

\subsection{Self-energy to 1'st order: $\Sigma^{(1)}$}

As Lüscher showed [1], the leading finite volume effects are captured in $\Sigma^{(1)}$ which can be summed up in closed form

$$
\Sigma^{(1)}=\frac{1}{2} \int \frac{d^{4} q}{(2 \pi)^{4}} \sum_{n=1}^{\infty} m(n) G_{0}(q) e^{i q_{1} \sqrt{n} L} \Gamma_{\pi \pi}(\hat{p}, q,-\hat{p},-q),
$$




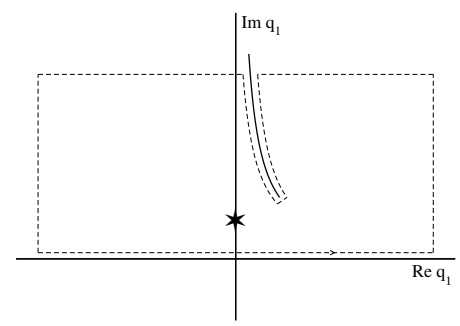

Figure 1: Integration contour in the complex $q_{1}$ plane (dashed line) with the pole from the pion propagator (diamond) and the branch cut from the $\pi \pi$-scattering amplitude.

with $\hat{p}=\left(i M_{\pi}, \mathbf{0}\right), \Gamma_{\pi \pi}(\hat{p}, q,-\hat{p},-q)$ the 4-point function of $\pi \pi$-scattering in the forward scattering kinematics and $m(n)$ the number of integer vectors $\mathbf{z}$ with $\mathbf{z}^{2}=n$. Eq. (3.4) may still be simplified considerably. We perform a contour integration in the complex plane of the first component $q_{1}$. Singularities are met by the pole of the propagator as well as the branch cuts from the propagator and the 4-point function. At the two-loop level only the cuts of the 4-point function have to be considered, lying on a hyperbola in the complex $q_{1}$ plane, as illustrated in fig. 1. Performing the contour integration along the dashed line, we obtain two terms, one from the residuum of the pole $I_{p}$ and the other from the integral along the new integration path. The latter contribution vanishes as we push the integration lines to infinity, except for the one along the cut, to be denoted by $I_{c}$ in the following,

$$
\Sigma^{(1)}=I_{p}+I_{c} .
$$

Along the lines discussed in [1], the former leads to the resummed asymptotic formula

$$
I_{p}=\frac{M_{\pi}^{2}}{16 \pi^{2} \lambda_{\pi}} \sum_{n=1}^{\infty} \frac{m(n)}{\sqrt{n}} \int_{-\infty}^{\infty} d y \mathscr{F}_{\pi}(\mathrm{i} y) e^{-\sqrt{n\left(1+y^{2}\right)} \lambda_{\pi}},
$$

with $\mathscr{F}_{\pi}(i y)$ the $\pi \pi$-forward-scattering amplitude. Restricting the sum to the first addend, we recover Lüscher's formula, eq. (1.1). Its extension to eq. (3.6) has already been suggested in [14, 16]. Numerically, the resummation turns out to be relevant for moderate $\lambda_{\pi}$.

Concerning the contributions of the cut $I_{c}$, we make use of a dispersive treatment. As these terms only start at the two-loop level, they are both suppressed in the chiral as well as in the large $L$ expansion and are therefore expected to be small. This is exactly what we observe numerically.

\subsection{Self-energy to 2'nd order: $\Sigma^{(2)}$}

Contributions from two pion propagators in finite volume are finally captured in $\Sigma^{(2)}$. Notice that the corresponding Feynman diagrams are uv-finite and need not to be renormalized. Therefore, it only remains to find a feasible numerical representation for these terms.

\section{Numerics}

The numerical analysis is performed in line with the setup of Ref.[16]. In fig. 2, we evaluate the relative finite volume shift

$$
R_{M_{\pi}} \equiv \frac{M_{\pi L}-M_{\pi}}{M_{\pi}}
$$




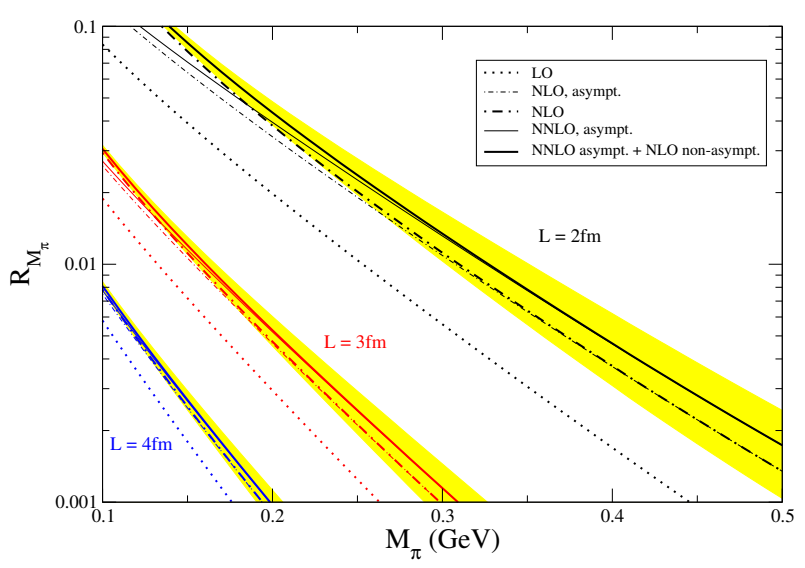

Figure 2: $R_{M_{\pi}}=M_{\pi L} / M_{\pi}-1$ vs. $M_{\pi}$ for $L=2,3,4 \mathrm{fm}$. For explanations of the legend, see text.

for $L=2,3,4 \mathrm{fm}$ as a function of $M_{\pi}$. We show the one-loop (LO) as well as the two-loop result (NLO). These shall be compared with the resummed asymptotic formula eq. (3.6) with LO/NLO/ NNLO input for the $\pi \pi$-scattering amplitude. Note that the one-loop result and the resummed asymptotic formula to LO agree with each other. The best estimate for $R_{M_{\pi}}$ is finally obtained by adding to the asymptotic pure three-loop contribution the two-loop result (NNLO asympt. + NLO non-asympt.). At NLO, the finite size effects encounter low energy constants, leading to a nonnegligible error band which is only shown for the best estimate. We take up a point already alluded in the introduction, namely the large contributions when going from LO to NLO in the asymptotic formula (dotted to thin-dash-dotted). Compared to this gap, the additional contributions from the full two-loop result (thick-dash-dotted) are very small. Consider eg. a pion mass of $M_{\pi}=250 \mathrm{MeV}$ in a $2 \mathrm{fm}$ box. We find $R_{M_{\pi}}=0.0236(41)$, of which 0.0010 stem from the two-loop corrections which are not included in the asymptotic formula. The two-loop and the NLO result from the asymptotic formula only drift away, when we go beyond the region where the $p$-regime can be safely applied.

\section{Conclusions}

We have evaluated the finite volume effects of the pion mass to two loops within ChPT in the $p$ regime. The results are compared with a recently proposed extension of the asymptotic formula of Lüscher. We find that contributions which were neglected in the latter, are numerically very small at the two-loop level and conclude that the resummed asymptotic formula is a convenient method to evaluate the finite volume effects beyond the leading order.

\section{Acknowledgements}

The work presented here is being done in collaboration with Gilberto Colangelo whom I warmly thank, also for a careful reading of the manuscript. This work is supported by the Swiss National Science Foundation and in part by RTN, BBW-Contract No. 01.0357 and EC-Contract HPRNCT2002-00311 (EURIDICE). 


\section{References}

[1] M. Luscher, Volume Dependence Of The Energy Spectrum In Massive Quantum Field Theories. 1. Stable Particle States, Commun. Math. Phys. 104, 177 (1986).

[2] J. Bijnens, G. Colangelo, G. Ecker, J. Gasser and M. E. Sainio, Pion pion scattering at low energy, Nucl. Phys. B 508, 263 (1997) [Erratum-ibid. B 517, 639 (1998)] [hep-ph/ 9707291$].$

[3] G. Colangelo, J. Gasser and H. Leutwyler, pi pi scattering, Nucl. Phys. B 603, 125 (2001) [hep-ph/0103088].

[4] G. Colangelo, S. Durr and R. Sommer, Finite size effects on M(pi) in QCD from chiral perturbation theory, Nucl. Phys. Proc. Suppl. 119, 254 (2003) [hep-lat/0209110]. G. Colangelo and S. Durr, The pion mass in finite volume, Eur. Phys. J. C 33, 543 (2004) [hep-lat/0311023].

[5] G. Colangelo and C. Haefeli, An asymptotic formula for the pion decay constant in a large volume, Phys. Lett. B 590, 258 (2004) [hep-lat/ 0403025$].$

[6] A. Ali Khan et al. [QCDSF-UKQCD Collaboration], The nucleon mass in $N(f)=2$ lattice QCD: Finite size effects from chiral perturbation theory, Nucl. Phys. B 689, 175 (2004) [hep-lat/0312030]. S. R. Beane, Nucleon masses and magnetic moments in a finite volume, Phys. Rev. D 70, 034507 (2004) [hep-lat/ 0403015$]$. S. R. Beane and M. J. Savage, Baryon axial charge in a finite volume, Phys. Rev. D 70, 074029 (2004) [hep-ph/ 0404131 ]. D. Becirevic and G. Villadoro, Impact of the finite volume effects on the chiral behavior of $f(K)$ and $B(K)$, Phys. Rev. $D$ 69, 054010 (2004) [hep-lat/0311028]. D. Arndt and C. J. D. Lin, Heavy meson chiral perturbation theory in finite volume, Phys. Rev. D 70, 014503 (2004) [hep-lat / 0403012 ].

[7] J. Bijnens, N. Danielsson, K. Ghorbani and T. Lahde, Two Loop Partially Quenched and Finite Volume Chiral Perturbation Theory Results, [hep-lat/0509042].

[8] A. Schenk, Pion propagation at finite temperature, Phys. Rev. D 47, 5138 (1993). D. Toublan, Pion dynamics at finite temperature, Phys. Rev. D 56, 5629 (1997) [hep-ph/9706273].

[9] G. Colangelo and G. Isidori, An introduction to CHPT, [hep-ph/ 0101264 ]. J. Gasser, Light-quark dynamics, [hep-ph/0312367]. S. Scherer and M. R. Schindler, A chiral perturbation theory primer, [hep-ph/0505265].

[10] U. G. Meissner, H. W. Hammer and A. Wirzba, Chiral Dynamics: Theory and Experiment (CD2003), [hep-ph/0311212].

[11] J. Gasser and H. Leutwyler, Light Quarks At Low Temperatures, Phys. Lett. B 184, 83 (1987).

[12] J. Gasser and H. Leutwyler, Thermodynamics Of Chiral Symmetry, Phys. Lett. B 188, 477 (1987).

[13] J. Gasser and H. Leutwyler, Spontaneously Broken Symmetries: Eeffective Lagrangians At Finite Volume, Nucl. Phys. B 307, 763 (1988).

[14] G. Colangelo, Finite volume effects in chiral perturbation theory, Nucl. Phys. Proc. Suppl. 140, 120 (2005) [hep-lat/0409111].

[15] G. Colangelo and C. Haefeli, to be published

[16] G. Colangelo, S. Durr and C. Haefeli, Finite volume effects for meson masses and decay constants, Nucl. Phys. B 721, 136 (2005) [hep-lat/ 0503014 ]. 